\title{
Arbor
}

\section{La construcción de la Torre de Babel}

\section{Manuel de las Casas Gómez}

Arbor CLXX, 671-672 (Noviembre-Diciembre 2001), 489-500 pp.

Al hablar de la conservación de la Ciudad histórica, de su uso o de su restauración, olvidamos con frecuencia de que ante todo es Ciudad, histórica o no y cometemos un gran error en las hipótesis de partida, de tal forma que la solución del problema, siguiendo el método científico, será errónea. Incluso aunque hablemos de la Ciudad como una pieza completa, que abarca desde el centro a la periferia y de la periferia al territorio una aproximación científica deductiva al problema basada es una visión objetiva del mismo, es solo válida para buscar soluciones concretas operativas, pero dudosamente válida para encontrar soluciones globales.

Y es que la Ciudad es mucho más que una connubación urbana, la Ciudad es sobre todo el lugar de los sueños de la humanidad, el lugar de los sueños que fueron, el lugar de las ensoñaciones presentes, el lugar de los sueños futuros (es en palabras de Savater «la sede la inmortalidad»). Es sobre todo el lugar de los afectos, de los recuerdos, el lugar de la memoria colectiva, y como la memoria está formada por múltiples capas superpuestas. Unas tan profundas como las que conservan el inconsciente colectivo, el «genio del lugar» evanescente y profundamente real, ¿existe esa Sevilla qué todos percibimos? Toledo, sede del más grande de los Imperios en su no despojada humildad ¿es un hecho mental o real? Otras, como las huellas de la traza, permanentes sobre los cambios sociales, estructurales o edificatorios, como cuando en un cuadro o en un dibujo por mucho que se borre o se enmascare siempre queda la huella de los trazos anteriores, como los recuerdos pasados, olvidados, pero presentes y conformadores de la Ciudad que conocemos. 
También las construcciones, existentes, pero desposeídas de sus significados primigenios, y los restos arquitectónicos de otros tiempos; unas como presencias físicas de lo que fueron formando parte del paisaje actual, otros convertidos en Monumentos de la memoria colectiva. Como esos recuerdos de la infancia; unos como esos héroes-hitos, otros como esa memoria o conocimiento mágico-simbólico de la realidad. Y por último, los objetos de uso avejentados pero no abandonados, los sustituidos, los de nueva traza, las nuevas conexiones urbanas, las nuevas puertas de la Ciudad, la Ciudad que vemos la que usamos, la que es igual a las demás con la casa, la oficina, el coche, el colegio, el poste de la luz, el hospital, el comercio, etc.

La Ciudad se asemeja a ese «baúl de la abuela», donde se almacena la ropa de uso diario con «aquellos zapatos que llevé a la boda de tu madre». Donde las cosas útiles y las ya no útiles se apilan unas sobre otras y cobran valor en esta mezcolanza, objetos que de vez en cuando hay que sacar, airear, reparar y de nuevo guardar, siempre añadiendo alguna nueva adquisición.

La ciudad solo puede entenderse, solo puede ser ella, en estas superposiciones, en este «umbral del caos» ¿Pero el «baúl de la abuela» es un caos?.

Una lectura aristotélica no vale para explicar el mundo en que vivimos. Los nuevos descubrimientos de las matemáticas, de la ciencia cierta, la matemática no euclidiana de los fractales, de las formas geométricas que están a punto de la descomposición que nunca ocurre, o las nuevas investigaciones con los ordenadores de las formas caóticas, que se repiten ordenadamente hasta el infinito, nos facilitan nuevas interpretaciones del universo que nos explican mas adecuadamente su complejidad.

La Ciudad es como el hombre que la creó, compleja, contradictoria, producto del azar y la necesidad, y está siempre como el hombre mismo en el «umbral» de algo, de ser lo que no es, de abandonar lo que fue, siempre inscrita en una continua renovación, porque junto al hombre, tiende a la inmortalidad.

La Ciudad es la nueva Torre de Babel, que intenta llegar hasta lo mas alto aunque por ello esté siempre al límite de su propia destrucción. Adjudicar a la Ciudad el papel que Rouseau da a la naturaleza, el lugar apartado idílico, es obviamente matar la Ciudad, es simplemente no entender lo que es la Ciudad.

Esto no significa abdicar a su conservación, sino entenderla desde un punto de vista nuevo, es como si al enfermar un brazo en lugar de curarlo aunque se deforme para que siga cumpliendo su papel, lo 
congelamos para que quede presente en nuestro aspecto aunque no sirva. Es creer más en los efectos que en los afectos y para nada vale conservar «efectivamente», conservar las apariencias, hay que conservar los afectos, los amores, los usos, aunque el brazo se deforme, no sea el más bello, ni el más musculoso.

$\mathrm{Y}$ para ello hay que amar y apoyarse en la singularidad, es como el símil del «baúl de la abuela». Hay que perseguir ese «genio del lugar» y solo esa loca carrera hacia lo desconocido, solo ese intenso riesgo de equivocarse, hace posible su recuperación.

Las directrices, las normas, las soluciones dadas en otros lugares, son correctivos que deben ser analizados al plantear las soluciones, pero incapaces una vez normalizadas y dogmatizadas de resolver las cuestiones. ¿Cómo una norma puede comprender el lenguaje poético de una ciudad? ¿Bajo que norma el Greco pudo completar la torre izquierda de la Catedral de Toledo? ¿O cómo es posible plantear la pirámide del Louvre sin entender el espíritu de grandeza que anima el nuevo crecimiento de París?

Analizar las presencias, es solo una parte del problema, hay que analizar las ausencias, como se recurre al Dios ausente para el entendimiento de nuestra existencia, como se investigan los agujeros negros del universo intentando comprender las «presencias» de hoy. Y esto es pensar en el futuro, no al modo de la ciencia ficción, sino de la única forma posible, proyectando el pasado mas profundo hacia los posibles caminos del futuro.

Así, con estas hipótesis de partida tendremos que analizar que mecanismos operativos se pueden usar para que las transformaciones, inherentes al hecho de ser Ciudad, no perturben este organismo, que fue artificial, como todos los hechos del hombre que completan o adecuan lo natural, transformándolo a conveniencia del uso pragmático o simbólico que cada sociedad requiere. Es el tiempo el que hace que lo artificial tome «carta de naturaleza» y que la Ciudad heredada se convierta en natural. $\mathrm{Al}$ actuar siempre lo hacemos a través de artificios, que si están adecuadamente planteados con el tiempo tomarán a su vez «carta de naturaleza».

Si analizamos con una mirada libre de prejuicios la historia de nuestras ciudades tendremos que reconocer que se formaron sobre otras ciudades distintas, probablemente útiles pero no adecuadas a los nuevos tiempos. Nuestras «Ciudades Eclesiásticas», son el resultado de las grandes construcciones religiosas, Iglesias y otros elementos de culto que transformaron entre el sigo XII y el XVI las ciudades existentes, creando otra nueva urbe que tenía no sólo que servir para habitar y 
comerciar, sino también para representarse a sí misma. Y en el XIX, el concepto de confort que desde la Inglaterra Isabelina se expande por el mundo, vuelve a producir grandes renovaciones urbanas adecuando las viejas ciudades a las nuevas formas de vivir y de entender nuestra existencia.

Así podemos llegar a la conclusión de que las ciudades son el resultado de múltiples transformaciones y adecuaciones al momento histórico y que para realizarlas se utilizan los «artificios modernos» de cada época.

¿Porqué entonces en nuestro siglo se produce un fuerte desacuerdo entre lo construido? La ciudad que se entiende como natural y los nuevos artificios que quieren renovarla.

En el siglo XX las viejas ciudades quisieron ser modernas, quisieron tomar un nuevo aspecto, parecerse a las de nueva planta, tener grandes edificios que representaran su capacidad comercial y social de las nuevas formas de vida. Un pequeño rascacielos era la aspiración de toda ciudad de provincia.

El mito del progreso que la revolución industrial plantea, «todo lo nuevo es mejor y lo viejo inservible» crea verdaderos problemas en la conservación de la Ciudad y la batalla poco fructífera establecida entre conservadores y progresistas poco ayuda a resolver los problemas que el crecimiento de las ciudades tienen. Este mito, es especialmente activo en las vanguardias arquitectónicas que como defensa de las nuevas propuestas no quieren saber nada de la Ciudad histórica que supone una pesada carga y solo la admiten como una Ciudad congelada a modo de museo.

Otro tanto ocurre desde la óptica de los historicistas y conservadores. Hasta el sigo XIX en el que la historia toma cuerpo científico después del estudio de la Antigüedad, realizado en las excavaciones de Roma, Egipto y Grecia, la historia se entendía como un devenir entre conservación, transformación y reutilización de las piezas arquitectónicas y sus materiales. Las cosas se valoraban en tanto en cuento servían, eran bellas o representaban algo para la sociedad del momento, el concepto de «monumento a conservar» como legado histórico independientemente de su valor de uso o simbólico no existía. Y así los conservadores caen en la misma equívoca lectura de la ciudad que los progresistas. Abogando por una ciudad que no transforme su imagen recurren a las arquitecturas mimétricas para resolver las inevitables transformaciones, o congelan la intervención en los edificios y en los espacios urbanos de tal modo que se provoca el abandono y por ende la ruina.

$\mathrm{Y}$ así como en el momento actual los progresistas reconocen el valor de uso de la Ciudad histórica, y el papel tan importante que 
en la creación y desarrollo urbano de las nuevas ciudades tienen los cascos consolidados como portadores de la memoria colectiva y de las señas de identidad, y que a través de la valoración de lo particular se puede llegar a lo universal, no lo tienen tan claro los conservadores, que no quieren admitir que preservar el pasado pasa necesariamente por la transformación del presente y que el problema de la conservación de la Ciudad histórica, no es un problema de estilo, sino de admitir que forma parte de un organismo más complejo que tiene que funcionar.

Visiones que se apoyen en la dicotomía centro-periferia, que entiendan que lo que no es casco histórico es crecimiento periférico y que la Ciudad está formada por dos ciudades; la histórica y la de nueva planta, no puede encontrar soluciones lógicas a la conservación de la primera. Solo entendiendo que el diseño de la nueva ciudad va a producir un efecto en la histórica y que si se cierran los ojos a la realidad los cascos históricos tendrán que soportar cargas de uso para los que no están preparados y provocarán su deterioro o abandono al ser sustituidos por otros nuevos centros podemos encontrar soluciones a su conservación.

Es necesario pues que la recuperación de los cascos esté inscrita en una estrategia global, léase planes generales o cualquier otra figura de planeamiento, que defina los usos de todas las partes y posibilite una Ciudad equilibrada que haga posible la reutilización de los cascos históricos. Cada caso será uno y son estos estudios los que deben definir las estrategias, es obvio que el ideal es una Ciudad completa donde residencia, comercio ocio, artesanía, negocios y edificios públicos convivan en un acuerdo único. Pero en cada ciudad en particular dadas sus estructuras edificadas, dada su topografía y sistemas de espacios públicos, dado su carácter de capitalidad o no, dada la presión turística, tendrá que buscar los equilibrios convenientes entre las partes. Y esto no es algo que surja solo, de forma natural, la Ciudad histórica tiende a la entropía, al abandono y a la degradación, y hay que tener una voluntad concreta y poner los medios necesarios para conseguir que estos cascos históricos ocupen el lugar que les corresponde, del que luego sus habitantes se sientan orgullosos y se conviertan en rentables para la Ciudad, para el uso de los ciudadanos y disfrute de los visitantes.

De las muchas cuestiones que habría que analizar, en mi condición de arquitecto voy a centrarme en una de ellas el CÓMO SE CONSTRUYE LA ARQUITECTURA.

Dos aspectos son fundamentales en la construcción de un edificio, el sistema constructivo y el tipo y tanto uno como otro en la construcción moderna se alejan de los usados en la historia, lo cual hace difícil el 
encuentro entre los edificios existentes con los de nueva traza. Analizar que sistemas constructivos son los adecuados en la ciudad y que tipos experimentados y depurados por la historia dan soluciones a la edificación, sin el uso irreflexivo de las nuevas maneras de hacer arquitectura, nos producirá un acercamiento profundo a la ciudad histórica, no la aproximación superficial que suponen las cuestiones de estilo.

Prácticamente desde el comienzo de la historia hasta este siglo, la arquitectura, con más precisión, la envolvente de los espacios arquitectónicos, se ha ejecutado por sistemas constructivos que Paricio llama «sistemas homogéneos» y que podríamos entender como sistemas no diferenciados. Sistemas donde un material único forma el muro de cerramiento, y este único material formado una masa continua sirve tanto de elemento estructural como de aislante térmico, acústico y de la humedad. A veces se llega a la continuidad total, y los techos aparecen resueltos por el sistema de bóvedas de diversos tipos, donde el mismo material resuelve a través de la geometría el problema de desplazar una carga vertical, desde un punto del espacio hasta otro horizontalmente, para así conseguir un espacio vacío.

La necesidad de apilar diversos pisos en el mismo espacio y las posibilidades que otros materiales ofrecen de trabajar a tracción, hace que se generalice el uso de la madera para resolver los tẹchos. Techos que pueden presentar volumen en cerchas o artesas, o que en búsqueda del mínimo espacio se convierten en planos. El muro sigue siendo homogéneo, y más o menos descargado o convertido en pórtico, exterior o interior, su comportamiento es el mismo. Su estabilidad, durabilidad y eficacia ante los agentes externos, la confiere fundamentalmente a la buena traba, a sus necesarios arriostramientos y a su adecuada geometría. Las impostas, jambas, alféizares, cornisas, acroterios, etc., es decir, todos los elementos que conforman la fachada, se deben a necesidades constructivas, fundamentalmente la de alejar el agua de la fachada, no dejando que discurra sobre grandes superficies, como son las cornisas o impostas. Y solucionar a través de la buena geometría la rápida evacuación en los puntos de concentración, como en jambas $\mathrm{y}$ alféizares y/o solucionar problemas de estabilidad del muro frente a las cargas que transmiten los diversos niveles de suelo sobre él y la apertura de huecos, o la propia estabilidad o durabilidad, en los puntos más expuestos como son las esquinas. Los diversos lenguajes hacen diferentes estos elementos pero siempre están presentes. Cuando los materiales son más homogéneos y más «duros», como las piedras o las cerámicas bien cocidas, muchos elementos pueden simplificarse hasta casi desaparecer; cuando, como en XIX y XX y ya a partir del 
barroco, con el uso de los revestimientos, los materiales son más «blandos», el diseño de estos elementos es de suma importancia, independientemente de ornamentación adicional cuya existencia y profusión es problema de otra índole.

La arquitectura, normalmente, está resuelta con materiales no «duros» incluso las piedras se comportan mal a tracción y en general ante la humedad, y desde luego, el barro, el adobe, el mampuesto, o las cerámicas poco cocidas, son materiales que necesitan de un fuerte cuidado en el diseño de sus elementos y en la mayoría de los casos además, capas protectoras como los revocos.

La cerámica bien cocida (sustituye en gran medida) en Europa del Norte a los demás materiales de construcción; su capacidad portante es muy alta. Su comportamiento ante los agentes externos, frío y calor es bueno; y para el agua sí es un elemento cargado. Por su pequeña dimensión es muy fácilmente manipulable y resuelve en una masa continua casi todos los problemas de borde, salvo los dinteles si no se introduce arco; con lo cual se pueden suprimir bastantes detalles constructivos que se resuelven de forma oculta en el plano; pero además es un material que puede formar muros suficientemente delgados en geometrías muy perfectas y permite la aparición del doble muro, de un muro especializado en la protección frente al agua y al frió y calor, el muro externo o muro pluvial que es ayudado por la cámara de aire que sirve de elemento de descompresión y aislante térmico, y el muro interior más grueso que sirve además de elemento portante.

Este muro pluvial que no lleva cargas permite ocultar de forma más homogénea los elementos constructivos que se ven desdoblados a este muro externo y al muro interior. Esto se traduce inmediatamente en la posibilidad del uso de lenguajes más simples, como puede verse en la casa georgiana, y posteriores desarrollos que dan lugar a estas arquitecturas más simples que buscan su traducción a otros materiales.

La cubierta es el otro elemento que junto al muro más conforma la imagen de la arquitectura. Desde el comienzo de la historia, la búsqueda está en desalojar lo más rápidamente el agua, vertiéndola lo más lejos posible del edificio o recogiéndola en los climas secos. Dos tipologías han sido fundamentales en el desarrollo histórico: la cubierta inclinada que abarca todo el área centro meridional y septentrional de Europa, y la terraza que se utiliza en el sur europeo y norte de África

Históricamente la terraza se usa sólo en climas secos, de lluvias torrenciales, donde está garantizado el desecamiento rápido de los materiales, además se produce en extensiones pequeñas, loteando fuer- 
temente la construcción para evitar los efectos de la dilatación y poderlas absorber en los planos verticales. Cuando hay que cubrir grandes superficies se llega a soluciones realmente hábiles como por ejemplo la del triforio de la Catedral de Toledo.

Cuando se intenta utilizar en las cubiertas planas sistemas más tecnológicos, como la cubierta catalana, sólo pequeñas edificaciones la soportan sin graves inconvenientes. Así las ciudades tienen una imagen característica, bien de cubiertas muy loteadas, o bien las cubiertas inclinadas que a través de la geometría resuelven con piezas pequeñas la rápida evacuación del agua, y que dependiendo de los climas y los materiales que se usen tiene una determinada imagen por sus pendientes y coloración.

La aparición de los materiales bituminosos en el mercado y los cauchos, más la voluntad del movimiento moderno de la simplicidad, de hacer que cada arquitectura sea emblemática de sus ideas, unido a su posible bajo coste, hacen que se utilicen indiscriminadamente las cubiertas planas, suprimiendo canalones, alféizares de remate, etc. en cualquier clima, en cualquier lugar, haciendo verdaderamente difícil el encuentro con la ciudad construida. Los problemas de durabilidad que plantean las cubiertas planas está haciendo reconsiderar su uso, posible sólo gracias al invento de la cubierta invertida. Y así la vuelta al uso de cubiertas inclinadas, ocultas o vistas, crea de nuevo, remates de coronación; también se está generalizando en algunos climas la doble cubierta plana con un espacio ventilado que recoge la antigua tipología de los sobrados.

Lo mismo ocurre con el muro. La arquitectura de materiales continuos parece obsoleta ante las nuevas exigencias de confort, y se buscan nuevas soluciones. Soluciones que van a ser factibles por la aparición de las estructuras portantes, primero metálicas y luego de hormigón. El muro puede perder su condición portante y quedar exclusivamente reducido a sus condiciones de aislante térmico, acústico y de la humedad. El peso ya no es un aliado puesto que al no sujetar nada puede reducirse de tamaño, y cuanto menos pese mejor para la estructura, y se comienza a buscar materiales que resuelvan los problemas específicos del agua, del sonido, del calor o frío. Se pasa de la arquitectura de sistemas continuos a la arquitectura de elementos especializados.

Evidentemente, esta ruptura con la tradición constructiva supuso una revolución histórica y produjo nuevos lenguajes, realmente alejados de aquellos con los que a lo largo de los siglos las ciudades se habían construido. Los nuevos sistemas constructivos de capas que se añaden a una estructura portante, pueden dar lugar a lenguajes muy diferentes 
a los usuales, hasta una abstracción total. Pero el problema no está en este sentido; muchos de los mejores edificios actuales urbanos en su pretendida neutralidad, se encuentran perfectamente con edificios adyacentes y completan su imagen.

El problema se plantea en dos aspectos. Primero que el sistema diferenciado admite una calidad pésima de construcción, creándose múltiples fisuras, humedades, puentes térmicos, etc., pero que no se cae. $\mathrm{Y}$ en segundo lugar porque admite cualquier absurdo que al proyectista se le ocurra. Dos cuestiones que no eran tan fácilmente posibles en los sistemas continuos, donde la composición aparecía en cierto sentido obligada, y la mala o equivocada construcción producía rápidas ruinas.

¿Pero esta revolución, esta arquitectura diferente, nos plantea mejores soluciones que la anterior, o por el contrario hay que volver a reconsiderar la construcción monomaterial como mejor?

Todos conocemos los problemas que los distintos coeficientes de dilatación y el distinto comportamiento de los materiales frente a los cambios de humedad, se producen en arquitectura diferenciada. Que sólo a base de tecnologías muy precisas y de un gran consumo energético son viables; mientras que la arquitectura de más masa y de masa más uniforme, que se comporta en forma homogénea frente a los cambios de humedad y temperatura y, que actúa eficazmente como aislamiento, acústico no plantea estos problemas. Y hoy frente a la crisis energética, el paro, el menor precio de ejecución, debe ser considerada, y de hecho esto está ocurriendo, como una solución alternativa a la construcción de la arquitectura. Su comportamiento a la estabilidad general es además mayor. El peso es el mejor aliado de la estabilidad en arquitectura. En los países más desarrollados de norte de Europa conviven las dos posturas: los sistemas homogéneos, y los discontinuos de alta tecnología que también resuelven el problema. Pero cada día se abandonan más los sistemas hoy normales en la construcción española.

Y esta arquitectura, la homogénea, lleva a lenguajes próximos a los históricos, el basamento y la coronación, los arquitrabes y las jambas, todos los elementos aparecen de una forma natural. El que algunas arquitecturas, como el postmoderno, utilicen además prestadamente los diseños de otros momentos históricos es otro problema; aunque difícilmente se puede superar una cornisa renacentista en su aspecto constructivo y de utilidad. Lo puro ornamental puede ser de otra forma y la arquitectura puede planearse nuevos lenguajes, pero siempre tendrá de común con la historia que tiene que resolver con materiales parecidos los mismo o similares problemas. Si además de resolver igualmente los problemas constructivos es más adecuada a la integración en la 
ciudad parece claro que, sólo cuando la lógica lleve al sistema constructivo diferenciado, será conveniente su utilización.

La discusión no está en los estilos, sino en la buena construcción, que es sin lugar a dudas la mejor garantía del hecho arquitectónico. El Seagran con su exquisita y potente modulación es más clásico, en muchos aspectos, que el último postmoderno; o las perfectas modulaciones de los edificios de Le Corbusier. Y en cambio cualquier persona, medianamente sensible, repudiará los mil ejemplos miméticos a los que estamos acostumbrados en nuestras ciudades, y lo hará no en tanto que dude de su adecuación o no, de si es mejor o no una arquitectura más moderno o más progresiva, sino por su falacia constructiva. Cualquier observador medianamente culto captará inmediatamente que las formas constructivas son aparentes; que una pretendida apariencia de masa está dejando visible una construcción de la peor calidad, muros que ocultan estructuras, balcones que no podrían sujetarse, aleros que tergiversan su función.

Desde este análisis una buena y coherente construcción es la mejor garantía de aproximación a la ciudad histórica, que durante siglos ha depurado los tipos y elementos constructivos, bien con la correcta aplicación de los sistemas constructivos altamente diferenciados, aprovechando los conocimientos y recursos del actual desarrollo industria o bien en la vuelta a los sistemas tradicionales, a la «firmitas» resuelta a través de una construcción de materiales continuos que dan lugar a un uso controlado con menos consumo de energía que conlleva unos estilos que en su nuevo sentido de la belleza se aproximan más a las arquitecturas históricas al dar lugar a unas arquitecturas más legibles, más duradera y de mayor riqueza expresiva.

Los problemas que el coche, o el camión de reparto, plantean en los cascos históricos no se le oculta a nadie; no sólo en sus vías, sino con la introducción del garaje aparcamiento en la edificación. Pero hay otros aspectos aparentemente más inocuos pero que su influencia en la transformación urbana ha sido decisiva, y que al afectar a propiedades individuales es de más difícil control. Lo colectivo siempre tiene una presión reguladora importante para que cambie, pero el uso del derecho individual es más difícilmente controlable. Quiero hacer referencia al invento del elevador mecánico y al control climático a base del consumo de energía.

El ascensor posibilita trasladarse sin esfuerzo a cualquier altura sobre la cota de la calle; el control venía dado en gran medida por la capacidad constructiva, y no era necesario encontrar nuevos sistemas porque subir a pie más de unas determinadas alturas era imposible, el elevador y las estructuras de acero y hormigón hacen posible el 
La construcción de la Torre de Babel

acceso a cualquier cota. Pero además el ascensor equilibra la importancia de las plantas. Los nombre de entresuelo, principal, primero, segundo y ático que hacen referencia a su importancia, debido a la comodidad de acceso, y que se traslada a la composición, desaparecen. El loteo amorfo es posible a ser todas las plantas igual de buenas. El ascensor posibilita también que la casa unifamiliar de 3 y más plantas —derivada de la casa gótica-, pueda dividirse en pisos independientes y romper el tejido tipológico.

El otro gran factor desencadenante de la transformación es el posible control climático, no a través de las formas, no a través de la geometría que dio lugar a tipos muy específicos, sino a través del consumo de energía. La calefacción sustituyendo a las chimeneas, y el aire acondicionado, hacen pensar que es innecesario el uso de los elaborados tipos que la historia había desarrollado para solucionar estos problemas; la casa patio, la casa torre, las dobles cubiertas, las tebaidas, los sobrados, los porticados, los aleros, cornisas, que servían para el control energético se abandonan.

Y digo que hacen pensar en el innecesario uso por varias razones. Parece absurdo no aprovechar, aunque se disponga de toda la energía, el diseño de la forma, para el control climático; es como si un diseñador de coches o aviones no tuviera en cuenta el coeficiente de penetración aerodinámica. Existen también problemas de índole física y psíquica que apoyan el uso mixto. De momento, así como el calor se puede generar sin ruido y sin vibraciones, la producción de frío nos lo hace soportar constantemente; y al estar necesariamente cerrados para crear un ambiente artificial, los cambios climatológicos, los olores, los ruidos que avisan de otros acontecimientos desaparecen. No es tan evidente su uso indiscriminado, cuando por medios naturales con el uso de materiales y geometrías adecuadas se puede resolver. Los climas artificiales tampoco pueden sustituir la emoción que uno siente en la contemplación de los grandes e inertes espacios abovedados, o la belleza espacial de patio que con sus velas y su naturaleza pretendidamente artificial nos habla de otras cosas, ni las escaleras con lucerna de la Casa Balear que con su diseño extrae el aire caliente del conjunto; espacios decantados por la historia por su capacidad para el control climático.

El mal uso de las nuevas conquistas tecnológicas y la confianza en que ellas por sí mismas iban a dar solución a una mejor Ciudad conduce a la reducción tipológica urbana y edificatoria que a partir del racionalismo se instaura en el crecimiento de las ciudades. La utilización de los nuevos tipos edificatorios sin la conversión a modelos concretos da lugar a las ciudades de hoy, abstractas por continuidades 


\section{Manuel de las Casas Gómez}

simples, que no cualifican el espacio, o por contigüidades descoordinadas espacialmente basadas en la trama del plano que nada tienen que ver con la imagen visual en la realidad. Solo baste a modo de ejemplo recordar las voces: rincón, calleja, callejón, corredera, travesía, calle, paseo, boulevar, avenida, costanilla, pasaje, rambla, camino, que nos hablaban de la forma, dimensión y cualidad de un espacio urbano lineal que hoy denominamos genéricamente calle o vía pública. Esta reducción en el lenguaje implica una reducción en el pensamiento, si no recuperamos el antiguo conocimiento del espacio y de la forma de construirlo difícilmente podremos actuar en la Ciudad histórica para conseguir su conservación y la mejora y aumento del legado para las futuras generaciones. 